\title{
A computer interview language: Programming the on-line interactive computer
}

\author{
HUGH V. ANGLE \\ Duke University Medical Center, Durham, North Carolina 27707 \\ and \\ JUDITH CARROLL \\ University of Wisconsin, Madison, Wisconsin 53706
}

\begin{abstract}
Seven computer interview and two disk file commands are executed by a computer program to display multiple-choice questions on a CRT video screen, to record clients' answers, and to branch to various sets of questions. The program interpreting these commands uses little computer memory and does not require change each time the interview is altered or expanded. It can be written in almost any general-purpose programming language.
\end{abstract}

Computer interviewing of psychiatric clients is a labor-saving technique (Coddington \& King, 1972; Johnson \& Williams, 1975), a way to improve the quality and reliability of interview information (Maultsby \& Slack, 1971; Stillman, Roth, Colby, \& Rosenbaum, 1969 ), and an evaluation procedure that is accepted and enjoyed by most clients (Angle, Ellinwood, \& Carroll, 1978; Slack \& Van Cura, 1968). Klingler, Miller, Johnson, and Williams (1977) have evaluated automated interviewing and found it to be a viable approach. The computer mechanics of interviewing, however, have received scant attention. As costs of computers continue to drop, more persons will be attracted to the possibilities of automated interviewing; their first practical concern will be programming the computer.

Investigators who have access to computers with specialized programming languages, such as computeraided instruction, or medical operating systems, such as MUMPS (Klein, Greist, \& Van Cura, 1975), have direct programming support for computer in terviewing. Most individuals, however, must contend with computers that are supported only by general programming languages. With a general language, such as FORTRAN, $\mathrm{PL} / 1$, or BASIC, one approach is to directly translate the interview questionnaire into the general language statements. This is the quickest way to create an interview system, but it has drawbacks: The interview program must be changed and recompiled each time a computer question is altered or added, and a lengthy interview may easily cause the interview program to exceed the size of the computer memory. A more efficient approach divides the interview into two systems: One is associated with the content of multiplechoice questions, and the other with the structure of these questions.

Multiple-choice questions have a fairly uniform, repetitious, and simple structure. Such questions contain an interrogative statement and a finite number of mutually exclusive options or answers. A program to process the structure of these questions uses little memory and, more importantly, does not require modification as the interview grows. Since the content of multiple-choice questions is of infinite variety, this information can be stored on a mass storage device, such as a magnetic disk memory, to allow for practically unlimited expansion of the interview. The stored disk information must, however, be written in a form of command code. This article describes a set of seven interview commands and two file commands, as well as a program to process these commands.

Greater attention is given to the details of the command code than to the details of the program, FRAME, that interprets these commands. The reason for this is that the current FRAME program is written in BASIC programming language for a PDP-11/40 computer with a resource sharing time system (RSTS) operating system. Other researchers may not have the same computer or the same operating system or employ the same programming language; therefore, their versions of the FRAME program will be different. However, the detailed description of the command code defines the input/output requirements of the FRAME program and should provide sufficient instructions so that programmers can adapt their computer resources to accomplish the same ends.

Hopefully, different versions of FRAME will adhere to the format of the present command code. This would ensure the compatibility of our system with other interview systems so that the whole or portions of our interview can be easily transported to other systems with a minimum of recoding effort. Currently, the field of computer interviewing employs no standard interview language to facilitate borrowing, using, or at least examining the computer interview work of others. The 
interview code of one computer system must be translated into the code of another. Vast quantities of patient information, greater than the amount presently gathered with pencil-and-paper procedures, can be collected with automated procedures (Angle et al., 1978), but little progress has been made toward developing a full battery of computer interview instruments. Since no one individual can undertake the development alone, the exchange of instruments is expected to be a fairly common and necessary practice in the field of computer interviewing.

\section{INTERVIEW COMMANDS}

In a sequential disk file, data records are accessed on the basis of successive physical position, similar to the sequential processing of magnetic tape records. Sequential files are employed in the present interview system to store multiple-choice questions in the form of computer code. The code is a series of one-line commands; each line begins with a particular command letter, that is, D, F, B, L, X, C, S. The FRAME program opens a command file, reads each line of code in sequence, and performs operations as determined by the command letters. Table 1 illustrates a multiplechoice question displayed on the CRT screen and the code to make this display possible. The following sections describe the function of each command.

\section{Display Text Command (D)}

The display command moves a line of text from the disk file to the CRT screen (screen size equals 24 rows and 80 character columns per row). The text following the letter D is inserted into 1 of 24 rows of the CRT screen. For example, a question concerning marital status can be coded in a single display command:

\section{ARE YOU MARRIED: $\quad$ ENTER 1=YES, OR 2=NO}

This text is displayed (minus the command letter) in the first row of the CRT screen, assuming no other display commands preceded it. As displayed, it presents a question to the client and directs the client to enter a CRT keyboard response of " 1 " for yes or " 2 " for no.

The marital status question can be given a more aesthetic appearence by a series of display commands:

$\begin{array}{cc}\text { D } & \\ \text { D } & \\ \text { D } & \text { ARE YOU MARRIED } \\ \text { D } & \text { 1. YES } \\ \text { D } & \text { 2. NO }\end{array}$

The FRAME program displays each line of text on successive rows of the CRT. The first two display commands without text serve as fillers to move the question toward the middle of the screen. Similarly, the indented text of the display command will position the question toward the center of the screen to make it easier to view.

A display command may contain three parameters to aid in positioning text on the CRT screen. The first parameter indicates the column location $(1.80)$ to begin the text display, the second parameter indicates the row location (1-24), and the third parameter indicates whether to single space or double space the lines of text. In the display command, the column and spacing values remain in effect for subsequent display commands lacking parameter specification or in effect until a clear operation occurs (see clear command). Each parameter is followed by a delimiter or slash " $/ . "$ The display command:

\section{20/10/2/"text"}

begins the display of "text" in Column 20 of Row 10 and the subsequent lines of text are double spaced.

The cursor of the CRT is a blinking light or underline character that functions in the interview to indicate to clients where their answers or responses will be echoed on the screen. In the case of multiple questions per CRT display, the cursor also serves to indicate the question being asked. The symbol "\#” in a display command

Table 1

Relationship of Command Code to the Display of a Computer Interview Question

\begin{tabular}{|c|c|c|c|c|}
\hline \multicolumn{2}{|c|}{ Screen Location } & \multirow[b]{2}{*}{ CRT Display } & \multirow[b]{2}{*}{ Command } & \multirow[b]{2}{*}{ Command Functions } \\
\hline Row & Column* & & & \\
\hline & & & $\begin{array}{l}\text { L FRAME22 } \\
\text { C }\end{array}$ & $\begin{array}{l}\text { Label } \\
\text { Clear }\end{array}$ \\
\hline 5 & 20 & ARE YOU SAD & D 20/5/1/ARE YOU SAD & Display \\
\hline 6 & 24 & () & D $24 /(\# 3)$ & \\
\hline 7 & 22 & 1. OFTEN & D $22 / 1$. OFTEN & \\
\hline 8 & 22 & 2. SOMETIMES & D 2. SOMETIMES & \\
\hline \multirow[t]{4}{*}{9} & 22 & 3. SELDOM & D 3. SELDOM & \\
\hline & & & F 1,155 & Field-data \\
\hline & & & & Execution \\
\hline & & & B FRAME45 $(1,155)<(3)$ & Branch \\
\hline
\end{tabular}


prescribes the placement of the cursor (the symbol \# is not displayed). Once the computer text of a question is displayed, the cursor moves to its designated screen location and the system halts to wait for the client's response (see $X$ command). The FRAME program computes the cursor position by counting the number of CRT rows already used and the number of columns between the symbol \# and the preceding command letter.

The value $\mathrm{n}$ following the cursor symbol indicates the range of answers contained in the question and is not displayed on the CRT:

$$
\mathrm{D} \# \mathrm{n}, \quad \text { where } \mathrm{n}=\text { "number of possible answers" }
$$

The client's CRT response is a value associated with a particular answer and cannot exceed the number to the right of the \# symbol. This feature functions to edit the client's answer. In the example of marital status, there are only two possible answers; therefore, the value following the symbol \# is 2. The FRAME program rejects a client's response greater than this value.

\section{Field Data Command (F)}

For each interview question, a response must be stored in a unique field location of the client's data file. The field command contains the coordinates of this location. In the present interview system, a client's data file is represented by 10512 -byte records. The field command indicates the record number (1-10) and the field number (1-512). For example,

$$
\text { F } 4,125
$$

instructs the FRAME program to place the client's response in Field 125 of Record 4.

The record number and field number of the field command are used by other commands to reference the client's previous response or answer. When used in other commands (e.g., branch, label, and set commands), the command letter $F$ is dropped and the record and field values are enclosed in parentheses, for example, $(4,125)$.

\section{Branch Command (B)}

In a sequential file, the lines of code are processed in serial order. A branch command cannot change this order, but it can suspend the processing of subsequent commands until a desired question is located in the file. When commands are read by FRAME but their executions are suspended, computer questions intervening between the branch command and the question being sought will not be displayed. In effect, the operation branches around a set of questions.

Two commands are needed for the branch operation: (1) a branch command and (2) a label command (e.g., L "labelname"). The branch command contains a name to search for, and the label command contains the name involved in the search. The branch command,

\section{B FIND-LABEL}

causes the FRAME program to read subsequent lines of code without performing their specified operations. Normal processing is reestablished when a label command is found with the label name "FIND-LABEL." The branch command in the above example contains no qualification, and thus the search-for-a-label-name routine is executed unconditionally.

A true-false statement is included in the branch command to allow conditional branching. A branch operation will be initiated when a true-false statement, involving the comparison of two values, is found to be true. Usually, the client's answer is denoted by a recordfield location and is compared $(=\rangle,,\langle,\langle\rangle)$ to the value of an expected answer. In the following example, the branch command functions to identify clients who have a value of 2 stored in $F 1,3$. The command branches these clients (females) to a question that has the label name FEMALE. If $(1,3)=(2)$ is true, that is, the client indicated she was female, then the search-fora-label-name routine is initiated. If a value of 1 is found to be stored in $(1,3)$, then the client is male and the branch operation is not performed. The program merely processes the subsequent lines of code dealing with male clients.

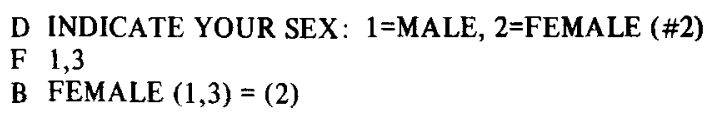

The branch command may also contain AND/OR conditions:

B FEMALE $(1,3)=(2)$ AND $(3,402)<(1)$ OR $(2,25)<>(4,510)$

\section{Label Command (L)}

A label name of the label command can be given to a group of code that constitutes an in terview question. A label command is essential when the question set is referenced by a branch command, and the name in the label command must match the name in the branch command. However, assigning a unique label name to every interview question delimits questions to facilitate management and organization of the file.

The true-false statement of the branch command can also be used in the label command. In a label command, it either causes the question to be skipped or to be displayed. If the true-false statement is true, then the subsequent lines of code are processed; if false, then the processing of commands is suspended until the next label command is encountered. The label processing logic (to execute code when a true condition is found) differs from that of the branch command. Furthermore, 
the label names in the label commands do not affect this processing. In the following code, female clients are presented the first question. Males are branched around this question and presented with the next question. Females are not presented the second question.

L "labelname" $(1,3)=(2)$

D HAVE YOU HAD A HYSTERECTOMY: $1=$ YES, $2=\mathrm{NO}(\# 2)$

L "labelname" $(1,3)=(1)$

D HAVE YOU HAD A VASECTOMY: $1=Y$ YE, $2=$ NO ( $\# 2)$

\section{Execution Command (X)}

The X-execution command causes the FRAME program to halt the processing of commands and to transfer computer control to the client. This command places the system into a response mode in order for the client to enter an answer. Each computer question requires an $\mathrm{X}$-execution command, although not necessarily in a one-to-one relationship (i.e., one $X$ execution command per question). For example, a single X-execution command can be used to present more than one question on the same CRT screen. In the following example, the text of three questions are displayed on the same screen before the program encounters an X-execution command.

D INDICATE $1=$ YES, $2=$ NO
D (\#2) ARE YOU SAD
D (\#2) ARE YOU NERVOUS
D $(\# 2)$ DO YOU LACK ENERGY
F 2,101
F 2,102
F 2,103
X

When the client answers the first question, the cursor moves to the second question, and so on. The FRAME program, however, must have the capacity to store the location of multiple cursor positions and record-field locations. In the above example, if an X-execution command is inserted after each question, then only the first question is displayed initially. When answered, the second question is displayed on the same screen, and so on.

\section{Clear Command (C)}

The clear command erases an entire screen of text and usually follows a label command to clear the text of the previous question so that new text can be displayed. The clear command also serves a housekeeping function to reset flags and variables in the FRAME program. For example, the row pointer of the FRAME program is initialized to one, so that the text of the first display command following the clear command is inserted into Row 1 of the CRT.

\section{Set Weight Command (S)}

Complex decision making is accomplished with the set command. This command employs the true-false statement to conditionally assign values to a variable or counter. The variable can subsequently be used in another true-false statement. FRAME contains 10 counters ( $\mathrm{CO}-\mathrm{C} 9$ ) that can be updated (added, subtracted, multiplied, divided), depending on the client's answers. The set command

$$
\text { S C } 3+4(1,3)=(2)
$$

adds a value of 4 to the content of counter C3, provided that the client is female, that is, a 2 is stored in $(1,3)$. For example, counter $\mathrm{C} 3$ may be updated by 1 every time the client gives a positive answer to a question concerning anxiety (a set command must be associated with each anxiety question). Later, the counter can be used in the true-false statement of a branch or a label command to present special interview questions to clients who have a certain level of anxiety.

$$
\mathrm{L}(\mathrm{C} 3)>(12)
$$

or

$$
\text { B ANXIETY-QUESTIONS (C3) > (12) }
$$

\section{File Commands}

Next-file command (N). Although the commands of an interview may reside in a single disk file, it is advisable to divide a lengthy interview into different files and assign a unique file name to each. This division facilitates management and flow of the interview. In the case of multiple files, information must be given at the end of the current file to define, by file name, the next file to be processed by FRAME. The following example of code illustrates the format of the next-file command. It also illustrates the branching to one of two disk files, depending on whether the client is male or female.

$$
\begin{aligned}
& \text { L }(1,3)=(2) \\
& \text { N MALE.FRM } \\
& \text { L }(1,3)=(1) \\
& \text { N FEMALE.FRM }
\end{aligned}
$$

Get program command (G). The computer interview language was developed for a 3,450-question problem screen and for a 1,000-question in-depth sexual survey. Over 700 psychiatric hospitalized and clinic patients have completed the problem screen. In the total interview, approximately $98 \%$ of the computer questions are handled by the FRAME program. The balance of the questions represent special CRT displays or special processing beyond the capabilities of the FRAME program. These questions are handled by separate interview programs. For example, after the FRAME program presents 50 fear questions and records the client's responses, another program, ORDER, selects only the fear items that the client indicated are intense fears and presents these items in a numbered list. The 
client is first asked to select the item of most concern and, following this choice, the item is erased from the list. The client is then asked to select the fear item of most concern from the remaining items, and this process repeats until only one item remains on the CRT screen. The ORDER program yields a hierarchy of fears or of any other problems assessed initially by FRAME.

To execute a special program during the interview, a get command calls the special program in to memory to replace the FRAME program. The get command contains the name of the program to be called (in a chaining operation) and the file name to be used when the program chains back to FRAME. The get command,

$$
\text { G PROG2.BAS (FILE4.FRM) }
$$

calls the program PROG2.BAS. When FRAME is reactivated, it opens the command file titled FILE4.FRM.

\section{FRAME PROGRAM}

The FRAME program begins with an instruction that (1) opens a sequential disk file. This instruction is followed by one that (2) reads a line of command code to extract the command letter. The command letter is used to transfer control to one of nine routines; each routine is associated with a particular command. For the most part, these routines involve the use of character string functions to decode the various commands. When the task of a routine is completed, control returns to Instruction 2 to read the next line of command code; however, the next-file and get routines are exceptions to this looping control. When the next-file command is encountered, the current file is closed, the name of the new file is extracted from the command, and control transfers to Instruction 1 to open the new file.

The overall size of FRAME is relatively small because many of the command routines share common functions and subroutines. The branch, label, and set routines employ the true-false statement and thus use the same subroutine functions, either to read data from the client's data file, or to compare two values in the true- false statement, or to AND/OR two or more sets of comparisons. The search-for-a-label operation of the branch routine is used elsewhere. First, it allows the client who has made a response error in the previous question to "back up," that is, to recover a question previously answered but erased. The label name(s) of the previous question(s) is always saved, and the client's input of a " $b$ " character causes FRAME to search for the saved label name of the earlier question (the current file is closed and reopened). Second, the search-for-a-label operation is used to restore the client at a restart point in the interview. When the client stops before completing the interview, the file name and label name are stored in the client's data file to be used in the restart operation.

\section{REFERENCES}

Angle, H. V., Elilinwood, E. H., \& Carroll, J. Computer interview problem assessment of psychiatric patients. Proceedings of the second annual symposium on computer applications in medical care. IEEE Computer Society, 1978.

Coddington, R. D., \& King, T. L. Automated history taking in child psychiatry. American Jouranl of Psychiatry, 1972, 129, 55-58.

Johnson, J. H., \& Williams, T. A. The use of on-line computer technology in a mental health admitting system. American Psychologist, 1975, 30, 388-390.

Klein, M. H., Greist, J. H., \& Van Cura, L. J. Computers and psychiatry: Promises to keep. Archives of General Psychiatry, 1975, 32, 837-842.

Klingler, D. E., Miller, D. A., Johnson, J. H., \& Williams, T. A. Process evaluation of an on-line computer-assisted unit for intake assessment of mental health patients. Behavior Research Methods \& Instrumentation, 1977, 9, 110-116.

MaUlTSBy, M. C., \& Slack, W. V. A computer based psychiatry history system. Archives of General Psychiatry, 1971, 32, 915.

Slack, W. F., \& V an Cura, L. J. Patient reaction to computer based medical interviewing. Computer and Biomedical Research, $1968,1,527-531$.

Stillyan, R., Roth, W. T., Colby, K. M., \& Rosenbaum, C. D. An online computer system for initial psychiatric inventory. American Journal of Psychiatry, 1969, 125, 8-11.

(Received for publication January 4, 1979; revision accepted March 30, 1979.) 